\title{
Influence of hydroxyethyl starch (6\% HES 130/0.4) administration on hematology and clinical chemistry parameters
}

\author{
Thomas Mueller ${ }^{1, *}$, Wolfgang Schimetta ${ }^{2}$, \\ Benjamin Dieplinger ${ }^{1}$, Peter Loeffler ${ }^{3}$, Markus \\ Rehm', Uwe Kreimeier", Werner Poelz ${ }^{2}$ and \\ Meinhard Haltmayer ${ }^{1,5}$ \\ ${ }^{1}$ Department of Laboratory Medicine, \\ Konventhospital Barmherzige Brueder, Linz, Austria \\ ${ }^{2}$ Department of Applied System Sciences and \\ Statistics, University of Linz, Linz, Austria \\ ${ }^{3}$ Department of Otorhinolaryngology, \\ Konventhospital Barmherzige Brueder, Linz, Austria \\ ${ }^{4}$ Clinic of Anesthesiology, Ludwig-Maximilians \\ University, Klinikum Grosshadern, Munich, Germany \\ ${ }^{5}$ Paracelsus Private Medical University, Salzburg, \\ Austria
}

\begin{abstract}
Background: The chemical inertness of hydroxyethyl starch (HES) might cause interferences of the colloid with a variety of laboratory tests. We aimed to evaluate potential influences of HES 130/0.4, the newest HES type, on several common hematology and clinical chemistry parameters.

Methods and results: A convenient sample of 25 patients scheduled for rheological therapy with $500 \mathrm{~mL} 6 \%$ HES 130/0.4 was evaluated. Blood samples were drawn before and after colloid application. Comparing pre- and post-infusion values of a battery of laboratory tests (i.e., hematology and hemostasis parameters, electrolytes, enzymes, kidney and metabolic parameters, lipids, etc.) in time course, a median difference greater than the reference change value for a specific parameter was considered clinically relevant. Among all parameters tested, only serum amylase activity displayed a clinically relevant difference between pre- and post-infusion values (median increase of $85 \%$ due to HES administration). By applying in vitro experiments, we demonstrated that serum amylase values obtained in the samples diluted in a $1: 1$ ratio with HES 130/0.4 and in samples diluted in a $1: 1$ ratio with $0.9 \% \mathrm{NaCl}$ displayed a negligible median difference of $3 \%$.
\end{abstract}

Conclusions: The in vivo effect of HES 130/0.4 administration on serum amylase activity observed in our

*Corresponding author: Thomas Mueller, MD, Department of Laboratory Medicine, Konventhospital Barmherzige Brueder, Seilerstaette 2-4, 4020 Linz, Austria

Phone: +43-732-7677-3621, Fax: +43-732-7677-3799,

E-mail: thomas.mueller@bs-lab.at

Received July 23, 2007; accepted January 2, 2008 study was pharmacological (real) in nature. With the exception of the influence of HES 130/0.4 on amylase activity, the effects of HES 130/0.4 on other parameters tested in this study can be interpreted as having no clinical relevance.

Clin Chem Lab Med 2008;46:558-62.

Keywords: drug effects; hemorheology; hetastarch; plasma substitutes.

Hydroxyethyl starch (HES) solutions are the most common plasma substitutes in Europe and more and more also in other parts of the world (1). The various types of HES differ in degree and position of hydroxyethylation and in average molecular weight (2). Some years ago, a newly designed third-generation HES type, called HES 130/0.4, was introduced into the market (2). HES 130/0.4 has improved elimination properties and optimized influences on the microcirculation compared to older HES types (3-5). As a consequence of the new pharmacological profile, the deterioration of coagulation, a well-known side effect of older HES types, has almost completely disappeared (6-14). Similar to all other HES types, HES $130 / 0.4$ also elevates serum amylase concentration $(9,12)$. This effect is explained by the generation of HES-amylase complexes impeding the renal excretion of amylase and, thus, inducing accumulation $(15,16)$. Besides the genuine influence on several laboratory parameters, HES is also able to influence the results of specific measurements (17-23). Although these observations were made for specific HES types only, it is possible that these effects may also occur in other HES preparations.

As the chemical inertness of HES might cause interferences of the colloid with a variety of laboratory tests in vivo and in vitro, we aimed to evaluate potential influences of HES 130/0.4, the newest HES type which is now already in widespread use, on several common hematology and clinical chemistry parameters.

The present single center study was performed prospectively at the St. John of God Hospital Linz, Austria. The study protocol was approved by the Local Ethics Committee in accordance with the Declaration of Helsinki, and written informed consent was obtained from all study participants. To determine possible changes in hematology and clinical chemistry parameters after application of HES 130/0.4, a convenient sample of 25 inpatients of the Department of 
Otorhinolaryngology scheduled for routine application of $500 \mathrm{~mL} 6 \%$ HES 130/0.4 in saline infusion $(0.9 \%$ $\mathrm{NaCl}$ ) was evaluated. Furthermore, 15 healthy volunteers (i.e., employees of the St. John of God Hospital Linz) receiving $500 \mathrm{~mL}$ saline infusion $(0.9 \% \mathrm{NaCl})$ served as controls. Exclusion criteria for the study participants were history of HES exposition, volume replacement therapy (e.g., dextrans, gelatins, albumin solutions) or blood transfusions (e.g., erythrocyte concentrates, fresh frozen plasma, platelets) within 2 weeks before enrolment into the present study, and the presence of any comorbidities (with the exception of arterial hypertension and diabetes mellitus).

The aim of the present study was to examine whether the presence of HES 130/0.4 in blood samples affects the results of several common hematology and clinical chemistry parameters. In case of an interference resulting in a "clinically relevant difference" between values obtained in a distinct parameter before and after HES 130/0.4 infusion, we additionally aimed to investigate by application of in vitro experiments (in vitro dilution of blood samples by HES $130 / 0.4$ vs. $0.9 \% \mathrm{NaCl}$ in a $1: 1$ ratio) whether this effect was masked (false) or pharmacological (real) in nature. The definition of a "clinically relevant difference" between values obtained in a distinct parameter before and after HES 130/0.4 infusion was based on the "reference change" concept for two successive measurements, as described later in the statistical methods section. The study medication was Voluven ${ }^{\circledR}$ (HES 130/0.4) 6\% infusion solution (Fresenius Kabi Austria $\mathrm{GmbH}$, Graz, Austria). The main physiochemical characteristics of Voluven ${ }^{\circledR}$ (HES 130/ $0.4) 6 \%$ infusion solution are as follows: average mean molecular weight of $130 \mathrm{kDa}$; molar substitution of $0.4 ; \mathrm{C} 2 / \mathrm{C} 6$ ratio of 9 ; HES concentration of $6 \%$; initial/distribution half-life $\sim 1.4 \mathrm{~h}$; and terminal/ elimination half-life $\sim 12 \mathrm{~h}(24)$.

Eligible individuals were asked to participate in the present study, and after appropriate information, they signed the consent forms. The first blood collection was carried out directly before HES 130/0.4 administration (in patients) or saline infusion (in controls). The duration of the infusion was $2-3 \mathrm{~h}$ by study design in both patients and controls. Approximately $2 \mathrm{~h}$ (range 1-3 h) after the end of the infusion, blood was collected from all study participants for the second time. All hematology and clinical chemistry parameters of interest for the present study (listed in Table 1) were determined from the blood samples obtained before and after the infusion. At each time point, the necessary amount of Vacuette erythrocyte sedimentation rate (ESR) sodium citrate, Vacuette EDTA K3, Vacuette $3.8 \%$ sodium citrate and Vacuette serum clot activator tubes (Greiner Bio-One, Kremsmuenster, Austria) were collected. Additionally, each parameter was determined in samples prepared by in vitro dilutions of the blood collected from the patients before HES 130/0.4 administration in a 1:1 ratio with Voluven $^{\circledR}$ (HES 130/0.4) 6\% infusion solution and saline infusion solution $(0.9 \% \mathrm{NaCl})$, respectively.

All laboratory analyses were performed within $2 \mathrm{~h}$ after blood collection by commercially available pro- cedures. ESR after $1 \mathrm{~h}$ was determined with a Sed Rate Screener 100 (Greiner Bio-One, Kremsmuenster, Austria); white blood cell counts, erythrocyte counts and platelet counts, as well as determinations of hemoglobin and mean cell volume were performed with a Cell-Dyn 4000 system (Abbott Laboratories, Abbott Park, IL, USA); prothrombin time according to Quick, activated partial thromboplastin time, and fibrinogen activity according to Clauss were measured on a BCS coagulation analyzer (Dade Behring, Marburg, Germany) with Dade Behring reagents (Thromborel S, Dade Actin FSL and Multifibren U, respectively); and all clinical chemistry parameters were assessed using serum samples by standardized methods on a COBAS Integra 700 analyzer (Roche Diagnostics, Mannheim, Germany). The following methods were applied: creatinine - kinetic Jaffe reaction without deproteinization (compensated method); blood urea - kinetic test with urease and glutamate dehydrogenase; sodium, potassium and chloride ion-selective electrodes; calcium - method according to Schwarzenbach with o-cresolphthalein; phosphate - direct phosphomolybdate method according to Daly and Ertingshausen (25); uric acid - enzymatic colorimetric test; bilirubin total - Diazo method; aspartate aminotransferase and alanine aminotransferase method with pyridoxal-5'-phosphate according to the International Federation of Clinical Chemistry and Laboratory Medicine (IFCC); $\gamma$-glutamyl transpeptidase - enzymatic colorimetric assay according to the IFCC; alkaline phosphatase - colorimetric assay according to the IFCC; lactate dehydrogenase - optimized standard method according to the German Society of Clinical Chemistry (Deutsche Gesellschaft für Klinische Chemie, DGKC); cholinesterase - method with S-butyrylthiocholine iodide; total creatine kinase (CK) - method according to the IFCC; creatine kinase muscle-brain type activity (CK-MB) - after immunoinhibition with antibodies to the CK-M subunit, the CK-B activity was determined with a method according to the IFCC; $\alpha$-amylase - enzymatic colorimetric assay according to the IFCC; lipase - enzymatic colorimetric assay with 1,2-O-dilauryl-rac-glycero-3glutaric acid-(6-methyl-resorufin) ester as substrate; glucose - enzymatic reference method with hexokinase; glycohemoglobin A1c ( $\mathrm{HbA} 1 \mathrm{c})$ - total hemoglobin and $\mathrm{HbA} 1 \mathrm{c}$ were determined after hemolysis of the anticoagulated blood specimen, total hemoglobin was measured colorimetrically, HbA1c was determined immunoturbidimetrically, the ratio of both concentrations yielded the final percent $\mathrm{HbA} 1 \mathrm{c}$ result; total cholesterol - enzymatic colorimetric method; high-density lipoprotein cholesterol and low-density lipoprotein cholesterol - homogeneous enzymatic colorimetric assays; triglycerides - enzymatic colorimetric method with glycerol phosphate oxidase and 4-aminophenazone; C-reactive protein - particle enhanced turbidimetric assay. HES serum concentrations were measured using the hexokinase method as previously described (26).

Statistical analysis was performed with the SPSS software release 13.0 (SPSS Inc., Chicago, IL, USA). 


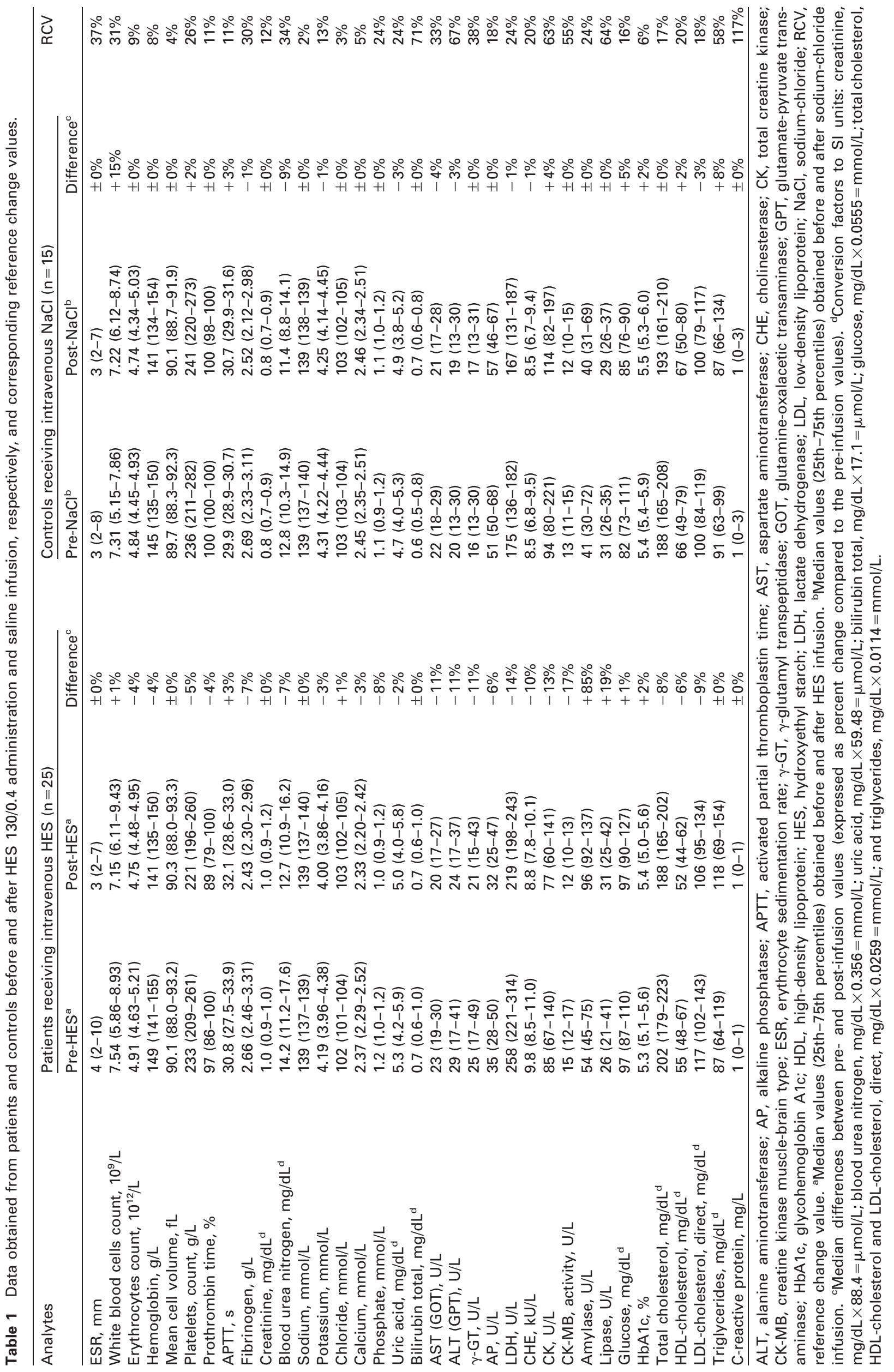


Data of hematology and clinical chemistry parameters were expressed as median (25th-75th percentiles). In patients with HES 130/0.4 administration and in controls with saline infusion, differences between preand post-infusion values were expressed as percent change of the post-infusion values compared with the pre-infusion values (applied for the data depicted in Table 1). According to the "reference change" concept for two successive measurements as mentioned previously, a "clinically relevant difference" of values obtained in a distinct parameter before and after HES $130 / 0.4$ infusion was evident by definition, if the median percent change of the post-infusion values compared with the pre-infusion values was greater than the reference change value (RCV) for a specific parameter. RCVs for each analyte are shown in Table 1 and were calculated as follows (27): $\mathrm{RCV}=\mathrm{z}_{\mathrm{p}} \times \sqrt{ } 2 \times \sqrt{ }\left(\mathrm{CV}_{\mathrm{W}}{ }^{2}+\mathrm{CV}_{\mathrm{A}}{ }^{2}\right)$, where $\mathrm{z}_{\mathrm{p}}$ is probability density function (generally 1.96 at $p=0.05$ of both side variations), $\mathrm{CV}_{\mathrm{W}}$ is within-subject biological variation and $\mathrm{CV}_{\mathrm{A}}$ is analytical variation of the test. $\mathrm{CV}_{\mathrm{W}}$ data were retrieved from the Westgard database (http://www.westgard.com/biodatabase1.htm, date of access 25 December 2007). Because no $\mathrm{CV}_{W}$ for ESR is listed in this database, we used a previously published value (28). $\mathrm{CV}_{\mathrm{A}}$ data were retrieved from the manufacturers' package inserts or product handbooks; in the case of more than one specification for a certain test, we used the lowest value given.

Of the 25 patients ( 20 male, 5 female; median age 38 years, range $21-56$ years) enrolled into the present study, 13 had acute hearing loss, 10 had tinnitus, one had vestibular syndrome and one had peripheral facial palsy. One patient had concomitant arterial hypertension and one had diabetes mellitus. The median duration of the HES 130/0.4 infusion was $2.4 \mathrm{~h}$ (range 2.1-2.9 h), and the median time interval between the end of the HES 130/0.4 infusion and the second blood withdrawal was $2.1 \mathrm{~h}$ (range 1.3-2.8 h). The median serum HES concentration at the second blood withdrawal was $0.20 \mathrm{~g} / \mathrm{dL}$ (range 0.14-0.31 $\mathrm{g} / \mathrm{dL}$ ). In the 15 healthy controls (10 male, 5 female; median age 35 years, range 21-52 years), the duration of the saline infusion and the time interval between the end of the saline infusion and the second blood withdrawal also complied with the study protocol.

In Table 1, the pre- and post-infusion values of each study parameter for both the patients and controls, as well as the median difference of the post-infusion values compared with the pre-infusion values and the RCVs are listed. In the patients, a median difference greater than the RCV was observed only for serum amylase. For this parameter, the median results obtained from samples prepared by in vitro dilutions of the blood collected from the patients before HES $130 / 0.4$ administration in a 1:1 ratio with Voluven ${ }^{\circledR}$ (HES 130/0.4) 6\% infusion solution and saline infusion solution $(0.9 \% \mathrm{NaCl})$, respectively, were $27 \mathrm{U} / \mathrm{L}$ (interquartile range $23-33$ ) and $27 \mathrm{U} / \mathrm{L}$ (interquartile range 23-38) with a median difference between those values (expressed as percent change compared to the $\mathrm{NaCl}$ values) of $3 \%$.
The main finding of the present study was that serum amylase activity was the only parameter among a battery of laboratory tests that was influenced in a clinically relevant manner due to the presence of HES 130/0.4 in vivo in the patients evaluated. Amylase activity increased by $85 \%$ in the blood samples obtained after intravenous administration of $500 \mathrm{~mL}$ Voluven ${ }^{\circledR}$ (HES 130/0.4) 6\% infusion solution compared with the blood samples collected before the colloid administration. As expected, in healthy controls receiving $500 \mathrm{~mL}$ saline infusion no relevant effect on amylase activity could be observed. Furthermore, by applying in vitro experiments we demonstrated that no relevant effect on serum amylase activity was detectable by the presence of HES 130/ 0.4 in diluted blood samples. Comparing the values obtained in the samples diluted in a 1:1 ratio with HES $130 / 0.4$ vs. the samples diluted in a $1: 1$ ratio with $0.9 \%$ $\mathrm{NaCl}$ (both derived from the 25 patients before the infusion HES 130/0.4), a negligible median difference of $3 \%$ was observed for this parameter.

Considering these issues, we conclude that the in vivo effects of HES 130/0.4 administration on amylase activity observed in our study was pharmacological (real) in nature. Our results argue, therefore, against an interference of HES 130/0.4 itself with the analytical procedure of this laboratory test. This conclusion is supported by the in vitro experiment, where only $3 \%$ variation was observed after a 1:1 dilution of the specimens with HES, which contrasts with the larger bias recorded ex vivo.

A major limitation, however, regarding the possibility of generalizing our study results is the fact that we used distinct laboratory methods for the present work as described above, and it is thus possible that using other platforms or other analytical procedures might yield other effects. Therefore, the reproduction of our results in other centers or by multicenter studies would argue for their validity.

The $4 \%$ decrease of hemoglobin and erythrocytes induced by the infusion of $500 \mathrm{~mL} 6 \%$ HES 130/0.4 indicates a volume expanding effect of approximately $200 \mathrm{~mL}$ at the time of the second examination $\sim 4.5 \mathrm{~h}$ after starting the colloid application. This is in accordance with the pharmacological properties of HES $130 / 0.4$ which suggest relevant volume efficacy of a $6 \%$ solution for some hours also in situations without initial volume deficit $(29,30)$. Bearing in mind the complex process of volume normalization after hypervolemic infusion (31), the HES serum concentrations obtained after administration of $500 \mathrm{~mL}$ Voluven ${ }^{\circledR}$ (HES 130/0.4) 6\% infusion solution in our study suggest a residual colloid amount of approximately onequarter of the infused $30 \mathrm{~g}$ at the time point of the second blood collection, thus being consistent with our above described clinical observations. As not all examined parameters can be considered to decrease linearly with plasma volume changes, we arbitrarily compared the values of the first and the second examination without dilution adjustment, which might be considered another limitation of the study. In this context, it is important to emphasize that the 
present evaluation was based on the single administration of $500 \mathrm{~mL} 6 \%$ HES 130/0.4 for the purpose of rheological therapy. Hence, the results obtained in this work may not be extrapolated in clinical situations where volume substitution is necessary (i.e., infusion of higher HES doses in intensive care or emergency medicine).

\section{References}

1. Treib J, Baron JF, Grauer MT, Strauss RG. An international view of hydroxyethyl starches. Intensive Care Med 1999;25:258-68.

2. Boldt J, Suttner S. Plasma substitutes. Minerva Anestesiol 2005;71:741-58.

3. Waitzinger J, Bepperling F, Pabst G, Opitz J. Hydroxyethyl starch (HES) [130/0.4], a new HES specification: pharmacokinetics and safety after multiple infusions of $10 \%$ solution in healthy volunteers. Drugs R D 2003; 4:149-57.

4. Leuschner J, Opitz J, Winkler A, Scharpf R, Bepperling $\mathrm{F}$. Tissue storage of $14 \mathrm{C}$-labelled hydroxyethyl starch (HES) 130/0.4 and HES 200/0.5 after repeated intravenous administration to rats. Drugs R D 2003:4:331-8.

5. StandI T, Burmeister MA, Schroeder F, Currlin E, Schulte am Esch J, Freitag M, et al. Hydroxyethyl starch (HES) $130 / 0.4$ provides larger and faster increases in tissue oxygen tension in comparison with prehemodilution values than HES $70 / 0.5$ or HES $200 / 0.5$ in volunteers undergoing acute normovolemic hemodilution. Anesth Analg 2003;96:936-43.

6. Jamnicki M, Zollinger A, Seifert B, Popovic D, Pasch T, Spahn DR. Compromised blood coagulation: an in vitro comparison of hydroxyethyl starch 130/0.4 and hydroxyethyl starch 200/0.5 using thrombelastography. Anesth Analg 1998;87:989-93.

7. Konrad CJ, MarkI TJ, Schuepfer GK, Schmeck J, Gerber HR. In vitro effects of different medium molecular hydroxyethyl starch solutions and lactated Ringer's solution on coagulation using SONOCLOT. Anesth Analg 2000;90:274-9.

8. Haisch G, Boldt J, Krebs C, Kumle B, Suttner S, Schulz A. The influence of intravascular volume therapy with a new hydroxyethyl starch preparation (6\% HES 130/0.4) on coagulation in patients undergoing major abdominal surgery. Anesth Analg 2001;92:565-71.

9. Langeron O, Doelberg M, Ang ET, Bonnet F, Capdevila $X$, Coriat P. Voluven, a lower substituted novel hydroxyethyl starch (HES 130/0.4), causes fewer effects on coagulation in major orthopedic surgery than HES 200/0.5. Anesth Analg 2001;92:855-62.

10. Franz A, Braunlich P, Gamsjager T, Felfernig M, Gustorff $B$, Kozek-Langenecker SA. The effects of hydroxyethyl starches of varying molecular weights on platelet function. Anesth Analg 2001;92:1402-7.

11. Boldt J, Haisch G, Suttner S, Kumle B, Schellhaass A. Effects of a new modified, balanced hydroxyethyl starch preparation (Hextend) on measures of coagulation. $\mathrm{Br} \mathrm{J}$ Anaesth 2002;89:722-8.

12. Woessner R, Grauer MT, Dieterich HJ, Bepperling F, Baus D, Kahles T, et al. Influence of a long-term, highdose volume therapy with $6 \%$ hydroxyethyl starch 130 / 0.4 or crystalloid solution on hemodynamics, rheology and hemostasis in patients with acute ischemic stroke. Results of a randomized, placebo-controlled, doubleblind study. Pathophysiol Haemost Thromb 2003;33: 121-6.
13. Neff TA, Doelberg M, Jungheinrich $C$, Sauerland A, Spahn DR, Stocker R. Repetitive large-dose infusion of the novel hydroxyethyl starch $130 / 0.4$ in patients with severe head injury. Anesth Analg 2003;96:1453-9.

14. Jungheinrich C, Sauermann W, Bepperling F, Vogt NH. Volume efficacy and reduced influence on measures of coagulation using hydroxyethyl starch 130/0.4 (6\%) with an optimised in vivo molecular weight in orthopaedic surgery: a randomised, double-blind study. Drugs R D 2004;5:1-9.

15. Kohler H, Kirch W, Weihrauch TR, Prellwitz W, Horstmann HJ. Macroamylasaemia after treatment with hydroxyethyl starch. Eur J Clin Invest 1977;7:205-11.

16. Mishler JM, Dürr HK. Macroamylasaemia following the infusion of low molecular weight-hydroxyethyl starch in man. Eur Surg Res 1979;11:217-22.

17. Kleine $\mathrm{N}$. The influence of the plasma expander hydroxyethyl starch on the serological blood grouping tests as compared to dextran 60 and whole blood. Anaesthesist 1975;24:225-7.

18. Daniels MJ, Strauss RG, Smith-Floss AM. Effects of hydroxyethyl starch on erythrocyte typing and blood crossmatching. Transfusion 1982;22:226-8.

19. Dieterich HJ, Neumeister B, Agildere A, Eltzschig HK. Effect of intravenous hydroxyethyl starch on the accuracy of measuring hemoglobin concentration. J Clin Anesth 2005;17:249-54.

20. Lagneau F, Beyne P, Letteron P, Laperche T, Marty J. Fluid therapy directly interferes with immunoassay for cardiac troponin I. Intensive Care Med 1999;25:625-7.

21. Millon L, Barale T, Julliot MC, Martinez J, Mantion G. Interference by hydroxyethyl starch used for vascular filling in latex agglutination test for cryptococcal antigen. J Clin Microbiol 1995;33:1917-9.

22. Hiippala ST. Dextran and hydroxyethyl starch interfere with fibrinogen assays. Blood Coagul Fibrinolysis 1995; 6:743-6.

23. Sommer R, Lapin A. Plasma expanders as cause of paraproteinuria-like artifact. Lancet 1991;337:499.

24. Jungheinrich C, Neff TA. Pharmacokinetics of hydroxyethyl starch. Clin Pharmacokinet 2005;44:681-99.

25. Daly JA, Ertingshausen G. Direct method for determining inorganic phosphate in serum with the "CentrifiChem". Clin Chem 1972;18:263-5.

26. Rehm M, Haller M, Orth V, Kreimeier U, Jacob M, Dressel $\mathrm{H}$, et al. Changes in blood volume and hematocrit during acute preoperative volume loading with $5 \%$ albumin or $6 \%$ hetastarch solutions in patients before radical hysterectomy. Anesthesiology 2001;95:849-56.

27. Guidi GC, Lippi G, Solero GP, Poli G, Plebani M. Managing transferability of laboratory data. Clin Chim Acta 2006;374:57-62.

28. Leppänen $E$, Dugué $B$. When to collect blood specimens: midmorning vs. fasting samples. Clin Chem 1998;44: 2537-42.

29. Waitzinger J, Bepperling F, Pabst G, Opitz J, Müller M, Francois Baron J. Pharmacokinetics and tolerability of a new hydroxyethyl starch (HES) specification [HES (130/ $0.4)$ ] after single-dose infusion of $6 \%$ or $10 \%$ solutions in healthy volunteers. Clin Drug Investig 1998;16:151-60.

30. Waitzinger J, Bepperling F, Pabst G, Opitz J, Fackelmayer A, Boldt J. Effect of a new hydroxyethyl starch (HES) specification [6\% HES (130/0.4)] on blood and plasma volumes after bleeding in 12 healthy male volunteers. Clin Drug Investig 1999;17:119-25.

31. Kohler $H$, Zschiedrich $H$, Clasen R, Linfante A, Gamm H. The effects of $500 \mathrm{ml} \mathrm{10 \%} \mathrm{hydroxyethyl} \mathrm{starch} \mathrm{200/0.5}$ and $10 \%$ dextran 40 on blood volume, colloid osmotic pressure and renal function in human volunteers. Anaesthesist 1982;31:61-7. 